\title{
Etude des relations trophiques entre le biotecton et les Naididae (Nais spp., Oligochaeta) : importance du carbone organique (C.O.T.)
}

\author{
J. Juget 1 \\ P. Riera ${ }^{2}$ \\ R. Schmelz 1
}

Mots clés : biotecton, Naididae, Nais, C.O.T., microphytes, bactéries, relations trophiques.

La biomasse des Naididae du genre Nais produite par voie asexuée (paratomie) est corrélée de façon positive et significative avec la charge en carbone organique (C.O.T.) du substrat nutritif exploité par ce type de consommateurs en divers milieux dulçaquicoles (qu'il s'agisse de périphyton croissant naturellement sur hydrophytes, d'extraits de périphyton cultivés in vitro ou de biotecton développé in situ sur substrats artificiels.

L'importance quantitative du C.O.T. en terme de rendement énergétique, trouve confirmation dans l'obtention d'un effet dépressif sur la productivité de populations de Nais spp. lorsque le rapport C.O.T. (substrat nutritif)/biomasse (Nais) chute en-dessous d'une valeur $\approx 4$ (effet de type densité-dépendant).

L'expérimentation in situ révèle, par contre, l'absence en règle générale de corrélation significative entre, d'une part, la biomasse et/ou la production des Naididae (Nais spp.) et, d'autre part, la biomasse et/ou la production des microphytes des milieux de culture (celles-ci étant estimées à partir de la charge en pigments chlorophylliens), quelle que soit la valeur prise par le rapport C.O.T./chlorophylle.

Cette absence de corrélation tient en partie au fait que des espèces opportunistes à régime mixte telles que la majorité des espèces du genre Nais se trouvent favorisées par le degré croissant de diversité du substrat nutritif dont l'une des formes d'expression est l'accroissement de la valeur du rapport C.O.T./chlorophylle. Elle s'explique en particulier par le fait que les espèces algivores exploitent également les bactéries associées aux microphytes, comme en témoigne la chute de productivité de populations de Nais spp. en présence de microphytes, principalement de diatomées, soumises en début d'incubation à l'injection d'antibiotiques.

A study of the trophic relationship between biotecton and Naididae (Nais spp., Oligochaeta) : importance of organic carbon

Keywords : biotecton, Naididae, Nais, T.O.C., microphytes, bacteria, trophic relations.

The biomass of asexual Naididae (Nais spp.) shows a significant positive correlation with the organic carbon weigth (T.O.C.) of nutritive substrates subject to various conditioning (periphyton growing naturally on hydrophytes, periphyton extracts cultivated in glass enclosures immersed in situ, biotecton growing in situ on artificial substrates).

The energy role of the T.O.C. is proved because values $\leqslant 4$ of the relationship between T.O.C. (nutritive substrate) and biomass (Nais spp.) produce a depressive effect on the growth rate of Nais populations (density dependent effect).

On the other hand, experimentation in situ reveals, as a rule, no significant correlation between the biomass and/or production of Nais spp. and the biomass and/or production of the microphytes (the latter calculated from the development of the chlorophyll pigments) whatever value the T.O.C. - chlorophyll relationship can reach. That is due partly to the opportunistic mixed diet in most species of the genus Nais, r-strategists favoured by increasing diversity of the nutritive substrate defined as increasing T.O.C./chlorophyll relationship. The lower growth rates of populations of Nais spp. cultivated on microphytes extracts sterilized with antibiotics, compared with control culture media without antibiotics, confirm their mixed diet viz. algivorous and bacteriophagous.

1. Laboratoire de Biologie Animale et Ecologie, URA CNRS 1451, « Ecologie des eaux douces et des grands fleuves », Université Claude Bernard (Lyon I), 43, Bd du 11 novembre 1918, 69622 Villeurbanne Cedex, France.

2. CREMA l'Houmeau (CNRS-IFREMER), 17317 Nieul-sur-Mer, France. 


\section{Introduction}

Au vu de leur abondance et de leur diversité, les Naididae représentent l'une des principales familles d'oligochètes limnicoles. La plupart des Naididae font figure de brouteurs de périphyton ou de biotecton. La contribution des algues unicellulaires, notamment des diatomées, dans leur alimentation a été notée par de nombreux auteurs ; la présence simultanée dans le bol alimentaire, chez de nombreuses espèces, de microphytes et de particules inertes organiques et/ou minérales suggère toutefois un régime mixte algivore - détritivore - limivore (Mc Elhone 1979, 1980, cité par Giani 1984, Provot 1982, in Juget 1984).

Le caractère opportuniste de l'alimentation et de la reproduction qui transparaît notamment au travers de leur comportement de brouteurs de biofilms et de leur forte productivité associée à la paratomie tend à assimiler les représentants de la famille des Naididae à des stratèges $r$ et exprime leur originalité par rapport aux autres familles d'oligochètes aquatiques, notamment les Tubificidae. Cette dernière remarque s'inspire en partie des résultats d'une étude des stratégies adoptées par les Oligochètes en fonction de leur affinité pour un type donné d'habitat, dans le cadre d'une recherche pluridisciplinaire visant à tester certains concepts écologiques en milieu fluvial (Juget \& Lafont 1994). C'est précisément à ce type de stratèges, en l'occurrence des populations de Naididae en phase de reproduction asexuée rattachées au genre Nais, qu'il est fait appel dans la présente étude, à titre de matériel biologique expérimental, pour analyser les relations trophiques entre producteurs et consommateurs de matières organiques à la surface de supports immergés naturels ou artificiels. Cette problématique privilégie le carbone organique (C.O.T.) en qualité de descripteur de la structure et du fonctionnement du réseau trophique producteurs-consommateurs de biotecton. Le rapport C.O.T./chlorophylle est perçu à cet égard comme une forme d'expression du degré de diversité du biotecton en qualité de substrat nutritif exploitable par les Naididae. Le rapport C.O.T. (biotecton) / biomasse (Nais spp.) permet, pour sa part, de tester les capacités trophiques du biotecton confrontées au concept écologique de densitédépendance.

Ce type d'approche repose sur un ensemble d'observations et d'expérimentations conduites principalement en étangs de pisciculture, au cours des récentes décennies. L'objectif initial de ces recherches consistait entre autres en l'étude d'impact de fertilisants sur la productivité des Naididae (Juget et al. 1989). Certaines informations inédites ainsi recueillies ont fait l'objet d'un traitement sélectif en conformité avec la problématique définie au paragraphe précédent.

\section{Matériel et méthodes}

\subsection{Sites d'étude}

Les observations et expériences conduites in situ correspondent à trois types de milieux, soit, par ordre chronologique, les étangs de la Réserve Biologique de Villars-lès-Dombes (Ain) $(1974,1989)$, les bassins de la Station expérimentale de l'Institut Régional de Recherches Appliquées en Aquaculture (IRRA) à Saint-Nizier-le-Désert (Ain) (1986, 1987), la rivière expérimentale et l'étang qui lui est associé, implantés sur le Campus de l'Université Claude Bernard (Lyon I) et dépendant du Laboratoire de Biologie Animale et Ecologie (1989, 1992).

\subsection{Protocoles expérimentaux}

L'expérimentation comporte deux types d'approche et de conditionnement.

\subsubsection{Culture in vitro sur substrats nutritifs inclus en enceintes de verre}

Ces enceintes constituées de piluliers de $125 \mathrm{ml}$ de contenance servent à confectionner un milieu de culture in vitro lequel est ensuite immergé in situ. Chaque enceinte abrite, en règle générale, $50 \mathrm{mg}$ d'extrait de biotecton de même provenance que les Naididae mis en incubation et obtenu par expression sous l'eau du périphyton de diverses hydrophytes puis filtration de l'extrait au travers d'un tamis de $0,07 \mathrm{~mm}$ de vide de maille. Le choix de la classe granulométrique $<0,07 \mathrm{~mm}$ a pour but de corriger l'effet dilutif dû à la présence éventuelle de particules grossières dans l'estimation de la charge en C.O.T. du substrat nutritif (exprimé en \% pondéral), dans la mesure où le diamètre moyen des plus grosses particules ingérables par les Oligochètes microdriles s'apparente à la taille de $0,07 \mathrm{~mm}$ (Juget 1979). L'extrait de périphyton, après calibration, est filtré sur membrane Nuclépore No80 et le refus du filtre dilué dans $100 \mathrm{ml}$ d'eau (préalablement filtrée sur Whatman $G F / C$ ) de provenance diverse selon le 
protocole expérimental adopté. Les enceintes sont immergées à une profondeur fixe d'une vingtaine de $\mathrm{cm}$ en début d'expérimentation dans l'étang d'où proviennent le périphyton et les Naididae qui font l'objet de l'étude.

\subsubsection{Evolution in situ du biotecton croissant sur substrats artificiels}

Le recours aux enceintes expérimentales permet l'étude des relations in vitro entre producteurs et consommateurs. Un autre type d'approche fait appel à des substrats artificiels maintenus en contact direct avec leur environnement naturel et permettant le suivi du biotecton en cours de formation. Il s'agit soit de lames de verre porte-objet de $76 \times 26 \mathrm{~mm}$ immergées verticalement et solidaires d'une grillesupport assurant la flottabilité des substrats (expérimentation en étang), soit de plaques de contreplaqué de $15 \times 15 \mathrm{~cm}$ ancrées à la surface du sédiment et recouvertes de toile émeri de texture variable (expérimentation en rivière).

Le protocole détaillé des plans expérimentaux adoptés dans les deux cas de figure (enceintes et substrats artificiels) sera précisé ultérieurement.

\subsection{Mesure du carbone organique (C.O.T.) et de la chlorophylle}

Le C.O.T. est mesuré à l'aide d'un analyseur Dohrmann DC-80 équipé d'un module d'analyse de carbone organique particulaire par traitement à $800^{\circ} \mathrm{C}$ d'échantillons solides ou de suspensions.

La chlorophylle $a$ et ses phéodérivés sont mesurés par spectrophotométrie selon la méthode de Lorenzen (1967) avant et après acidification de l'extrait hydroacétonique (concentration finale de HCL dans l'extrait : 0,003 mole $\mathbf{1}^{-1}$; durée minimum du traitement à l'acide : 20' (Aminot 1983).

\subsection{Stérilisation du périphyton}

L'un des plans expérimentaux (cf. 3-5) avait pour objectif l'étude d'impact des bactéries associées au phytopériphyton sur la production des brouteurs algivores. Une partie des enceintes abritant les milieux de culture est stérilisée, au départ de l'expérience, par adjonction d'antibiotiques, sous la forme d'un mélange de Pénicilline $\mathrm{G}\left(10 \mathrm{mg} \mathrm{l}^{-1}\right)$ et de Streptomycine-sulfate $\left(20 \mathrm{mg} \mathrm{l}^{-1}\right)$, soit une concentration finale dans le milieu de culture de $0,003 \%$. La nature et la concentration des antibiotiques utilisés tiennent compte d'une part du fait qu'ils protègent les diatomées, élément prédominant du périphyton, d'effets secondaires algicides (Berland \& Maestrini 1969) et d'autre part, du fait qu'ils ne perturbent pas la croissance des Naididae (Provot, 1982).

Le contrôle de l'absence de bactéries dans les enceintes soumises au traitement aux antibiotiques, en début d'expérience, est assuré à l'aide d'un échan. tillonneur Total-Count de Millipore, l'abondance des bactéries dans les enceintes témoins étant estimée à $10^{5} \mathrm{ml}^{-1}$.

\subsection{Mesure de la biomasse des Naididae}

La biomasse individuelle (exprimée en poids sec) est calculée à partir de la mesure du diamètre moyen, de la longueur $\mathrm{L}$ et du biovolume selon la formule d'Abrahamsen (1973), en assimilant le corps de l'animal à un cylindre, sur des spécimens préalablement fixés au formol à $4 \%$ et montés entre lame et lamelle dans un liquide visqueux (Sirop d'Apathy) constitué d'un mélange de poudre d'acacia, de lévulose et d'eau distillée. Le facteur de conversion $\times 0,2$ est utilisé pour convertir le poids frais en poids sec. Le choix de ce facteur de conversion fait suite à un test de contrôle consistant en la double pesée avant et après passage à l'étuve à dessication d'un échantillon de 200 individus asexués de Nais spp. en provenance de l'étang Petit Turlet (Réserve Biologique de Villars-lès-Dombes), préalablement fixés au formol à $4 \%$.

\section{Résultats}

\subsection{Le périphyton des herbiers à Trapa natans, un substrat nutritif naturel exploité par les Naididae}

Le couvert végétal de ce type d'herbier qui contribue à modifier les caractéristiques physiques et chimiques des plans d'eau envahis par cette hydrophyte (Juget \& Rostan 1973) est constitué de rosettes flottantes d'un diamètre pouvant atteindre une trentaine de $\mathrm{cm}$ et dont le biovolume moyen circonscrit aux $5 \mathrm{~cm}$ superficiels peut représenter en période estivale plus des deux tiers du volume total de l'herbier.

Un inventaire du zoopériphyton inféodé à ce type d'herbier à été réalisé en Août-Septembre 1974, dans l'un des étangs de la Réserve Biologique de Villarslès-Dombes, l'étang Sainte-Anne, d'une superficie de 7 ha. Cet inventaire a révélé la prédominance de 
Nais variabilis dont les effectifs représentaient en moyenne $54 \%$ des peuplements de Naididae hébergés par les rosettes flottantes, soit un effectif moyen de 340 individus/rosette. Une étude des relations entre la charge en C.O.T. du périphyton (< $0,07 \mathrm{~mm}$ ) et la biomasse (poids sec) des Naididae du genre Nais révèle un coefficient de corrélation linéaire élevé ( $\mathrm{r}=0,843, \mathrm{P}<0,001, \mathrm{n}=10)$, en ce qui concerne le périphyton inféodé aux rosettes flottantes de l'herbier. La valeur moyenne de la charge en C.O.T. du périphyton (et son intervalle de confiance correspondant au coefficient de sécurité $95 \%$ ) s'élève à $11,8 \pm 6,92 \mathrm{mg} /$ rosette et celle de la biomasse correspondant au genre Nais à 7,38 $\pm 4,05 \mathrm{mg}$ (poids sec)/rosette. Quant à la valeur moyenne du rapport C.O.T. (périphyton)/biomasse (Nais), elle s'établit à 2,06 $\pm 1,16$.

\subsection{Mesure in vitro en conditions expérimentales semi-naturelles de l'impact du périphyton sur la structure et la dynamique des populations de Naididae}

Les données précédentes tirées de l'observation in situ en conditions naturelles laissent présager le rôle du C.O.T. en qualité de paramètre régulateur de la dynamique des populations de Naididae. Elles peuvent être confrontées aux résultats obtenus dans le cadre de recherches conduites au niveau des étangs de la Station expérimentale de l'IRRA à Saint-
Nizier-le-Désert (Juget et al. 1989 ; Riera et al. 1991).

Une expérience conduite du 10/07 au 10/08/1987 a consisté en un suivi de populations de Nais christinae Kasprzak, Nais barbata Müller, Nais communis Piguet, Nais simplex Piguet et Nais pardalis Piguet, provenant d'un stock initial de même origine de 10 individus triés au hasard et incubés en enceintes de verre, selon le protocole décrit en 2.2.1. L'eau introduite dans chaque enceinte est de même provenance que les populations de Naididae expérimentées, à savoir un étang non fertilisé, servant par ailleurs de témoin. Cette eau est renouvelée (enceintes $A$, cf tableau 1) ou non renouvelée (enceintes A') à l'issue de chaque tranche de temps d'une dizaine de jours chacune et reçoit en 3 fois consécutives (A) ou en une seule fois ( $\left.\mathrm{A}^{\prime}\right)$ une injection de superphosphate sous forme d'engrais liquide équivalente à $10\left(\mathrm{~A}, \mathrm{~A}^{\prime}{ }_{10}\right), 30\left(\mathrm{~A}, \mathrm{~A}^{\prime}{ }_{30}\right)$ ou 50 $\left(A, A^{\prime}{ }_{50}\right) \mathrm{kg} / \mathrm{ha} /$ an de $\mathrm{P}_{2} \mathrm{O}_{5}$, les enceintes $\mathrm{A}_{0}$ et $\mathrm{A}^{\prime}{ }_{0}$ non fertilisées servant de témoins. La dose totale de fertilisant est calculée en fonction de la capacité des enceintes et des temps d'incubation par référence à une campagne annuelle de fertilisation des étangs de la Station expérimentale.

Le tableau 1 indique les taux de recrutement cumulés des populations de Nais spp. introduites dans chaque enceinte et calculés en fonction du

Tableau 1. Relation entre les taux de recrutement cumulés de Nais spp. et les taux d'accroissement du C.O.T. et des pigments chlorophylliens (chlorophylle $a+$ phéodérivés) des milieux de culture. Station expérimentale (IRRA) ; expérience du 10/07 au 10/08/1987.

Table 1. Cumulative recruitment rates of Nais spp. and rates of increase of T.O.C. and chlorophyll (chlorophyll $a$ and pheopigments a) of the culture media.

Experimental station (IRRA) : experiment from July 10 to August 10, 1987.

\begin{tabular}{cccc}
\hline $\begin{array}{c}\text { Enceintes } \\
\text { (conditionnements) }\end{array}$ & $\begin{array}{c}\text { Taux de recrutement Taux d'augmentation } \\
\text { cumulés (Nais spp) } \\
\text { du C.O.T. }\end{array}$ & $\begin{array}{c}\text { Taux d'augmentation des } \\
\text { pigments chlorophylliens } \\
\text { (chlorophylle a + phéodérivés) }\end{array}$ \\
\hline A0 & 8,1 & 0,83 & 1,85 \\
A'0 & 6,6 & 0,37 & 1,71 \\
& & & \\
A10 & 9,5 & 1,18 & 2,57 \\
A'10 & 7,6 & 1,08 & 2,14 \\
A30 & 6,6 & 1,02 & 2,35 \\
A'30 & 6,4 & 1,27 & 3,50 \\
A50 & 8,2 & 1,37 & 3,14 \\
A'50 & 9,4 & 1,90 & 3,21 \\
\hline
\end{tabular}


nombre d'individus néoformés pour l'ensemble des trois étapes successives du plan expérimental (10/07-20/07, 20/07-30/07, 30/07-10/08/1987), le repiquage au hasard de 10 individus intervenant dans chaque enceinte à l'issue de chacune des étapes. $\mathrm{Ce}$ tableau précise également les taux d'accroissement de la charge en C.O.T. et en chlorophylle (chlorophylle $a+$ phéodérivés) des substrats nutritifs.

Une analyse de variance à 2 facteurs contrôlés (taux de fertilisation et mode de conditionnement hydraulique) [eau des enceintes renouvelée ou non renouvelée], (Lellouch \& Lazar 1974) a révélé l'absence de différences significatives dans la production cumulée des populations de Naididae du 10/07 au $10 / 08 / 1987$, quels que soient les taux et les modes de fertilisation en 1 ou 3 injections successives (Juget et al. 1989). Le calcul du coefficient de corrélation entre la production des Naididae, exprimée en terme de recrutement cumulé $\left(R_{N}\right)$ et la production des microphytes in vitro, calculée par le biais du taux d'accroissement en pigments chlorophylliens des enceintes entre le 10/07 et le 10/08/1987 $(r=0,195)$ confirme l'absence de relation significative entre ces 2 variables. En revanche, la production de microphytes estimée à partir du taux d'augmentation des pigments chlorophylliens, est impliquée de manière significative $(r=0,818, P<0,01)$ dans l'accroissement de la charge en C.O.T. du périphyton, tributaire de l'apport de fertilisants.

Conformément aux résultats obtenus sur l'étang Sainte-Anne (cf. 3.1.), la biomasse des populations de Nais spp est corrélée de manière très significative en fin d'expérience $(\mathrm{r}=0,923, \mathrm{p}<0,001)$ avec le C.O.T. du périphyton, la valeur moyenne du rapport C.O.T. (périphyton)/biomasse (Nais), chacun des 2 paramètres étant exprimé en $\mathrm{mg} / \mathrm{g}$, s'établissant à $10,63 \pm 5,15$ pour une charge moyenne en C.O.T. (périphyton) de $83,4 \pm 12,2 \mathrm{mg} / \mathrm{g}$ et une biomasse moyenne (poids sec) de Naididae de 10,53 $\pm 6,85 \mathrm{mg} / \mathrm{g}$.

\subsection{Mise en évidence en culture in vitro d'un effet de type densité-dépendant}

Les écarts importants constatés en ce qui concerne les rapports C.O.T. (substrats nutritifs)/biomasse (Naididae) in situ (cf 3.1) et in vitro (cf. 3.2), compte tenu du fait que ces 2 paramètres sont, dans les 2 cas de figure analysés, fortement corrélés, conduisent à s'interroger sur la signification de ces écarts et à suspecter dans le cas de rapports de faibles valeurs l'intervention d'un processus de régulation biologique de type densité-dépendant.

La démonstration d'un effet de ce type nous est fournie par l'analyse des résultats d'un plan d'expérience analogue au précédent et réalisé en 1986 du 13/06 au 21/07 dans le cadre de la Station expérimentale de l'IRRA. Le périphyton et les Naididae mis en incubation ont la même origine (étang non fertilisé) que dans l'expérience décrite en 3.2. et sont répartis entre 12 enceintes soumises à 2 types de conditionnement :

- Un conditionnement biologique comportant pour un tiers des enceintes le repiquage au hasard de 10 individus de Nais spp, à l'issue de chacune des 4 étapes successives d'une dizaine de jours chacune (conditionnement a), alors que pour les 8 autres enceintes (conditionnement b), la totalité des individus est remise en élevage après contrôle des effectifs à l'issue de chaque étape.

- Un conditionnement hydraulique défini dans cette expérience par la composition de l'eau introduite dans chaque enceinte en provenance de 4 étangs soumis à 4 modes distincts de fertilisation (un étang soumis à une fertilisation organique, un étang soumis à une fertilisation minérale, un étang soumis à une fertilisation mixte, un étang non fertilisé). Les 8 enceintes soumises au conditionnement biologique (b) défini plus haut sont réparties en 2 séries de 4 enceintes équivalentes à 2 séries répétitives.

La figure 1 exprime graphiquement l'évolution des taux moyens de recrutement cumulés et leur intervalle de confiance respectivement pour les 4 enceintes renfermant des populations de Nais spp soumises au repiquage de 10 individus (conditionnement a) et pour les 2 séries de 4 enceintes soumises au conditionnement (b), ces dernières étant traitées à titre d'essais répétitifs, l'analyse de variance ne révélant aucune différence significative des taux de recrutement cumulés entre les enceintes en fonction de leur conditionnement hydraulique.

Cette figure appelle plusieurs commentaires :

- Il n'existe aucune différence significative entre les lots de Naididae à l'issue de la $1^{\text {ere }}$ étape du 13/06 au $23 / 06$, quelles que soient l'origine et la nature de l'eau introduite dans les enceintes, l'ensemble des 12 enceintes étant soumis initialement au même type de conditionnement biologique. 


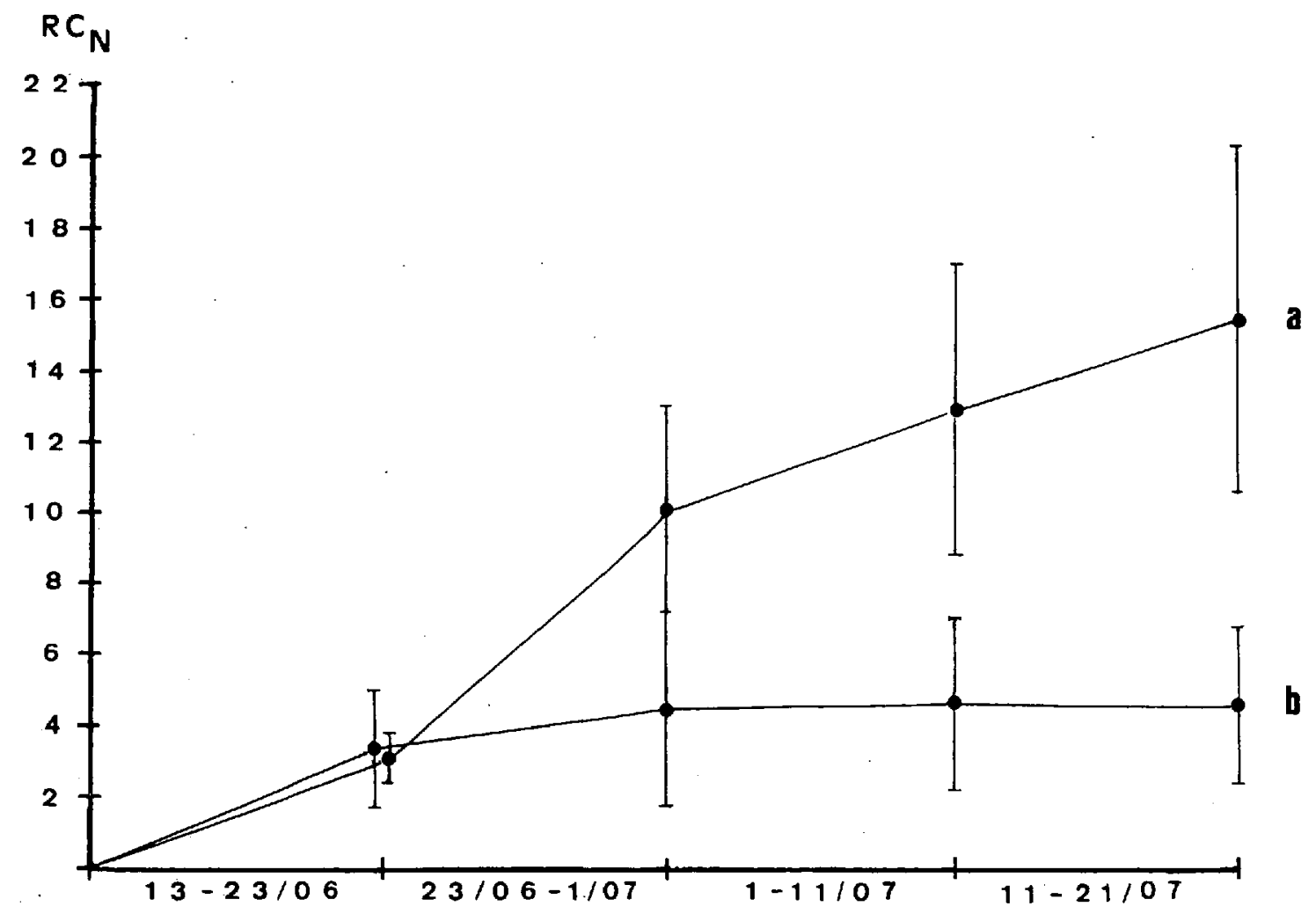

Fig. 1. Evolution comparée des taux de recrutement cumulés $\left(\mathrm{RC}_{\mathrm{N}}\right)$ de Nais spp. (valeurs moyennes et leur intervalle de confiance pour une sécurité de $95 \%$ ). Station expérimentale (IRRA) ; expérience du 13/06 au 21/07/1986.

a : enceintes avec Naididae soumises au repiquage.

$\mathrm{b}$ : enceintes avec Naididae non soumises au repiquage.

Fig. 1. : Cumulative recruitment rates of Nais spp. (mean values and $95 \%$ confidence limits). Experimental station (IRRA) ; experiment from June 13 to July $21,1986$.

a : enclosures with Naididae pricket out.

b : enclosures with Naididae without pricking out.

- Un plafonnement des taux de recrutement, associé accessoirement à une mortalité des effectifs, intervient dès l'issue de la $2^{\mathrm{e}}$ étape de l'expérimentation dans les enceintes soumises au conditionnement (b).

- Ce plafonnement coïncide avec des valeurs du rapport C.O.T. (substrat nutritif)/biomasse (Nais) inférieures aux limites de confiance de la moyenne calculées en 3.2 et proches de celles mentionnées en 3.1 , soit des valeurs $\leqslant 4$.

- Une partie ou la totalité des effectifs de $N$. communis et de $N$. simplex en cas de surpopulation atteignant ou dépassant une centaine d'individus par enceinte étaient sexuellement matures. Cette substitution de la reproduction sexuée à la reproduction asexuée par paratomie peut s'interpréter comme l'équivalent de la substitution chez les 2 espèces concernées d'une stratégie $\mathrm{k}$ à la stratégie $\mathrm{r}$, en réponse à un stress physiologique lié au surpeuplement (Loden 1981). Cette observation confirme l'existence de relations de densité-dépendance.

3.4. Recherches expérimentales sur le biotecton impliquant l'immersion in situ de substrats artificiels.

Le suivi du biotecton croissant sur divers types de supports permet de cerner notamment la dynamique des interrelations entre producteurs et consommateurs primaires. 
3.4.1. Les données du tableau 2 concernent une expérience conduite du 10/05 au 5/06/1989 sur l'étang Petit Turlet, étang piscicole de 30 ha de la Réserve Biologique de Villars-lès-Dombes, contigu à l'étang Sainte-Anne (cf. 3.1). Trois séries de lames de verre porte-objet de $76 \times 26 \mathrm{~mm}$ sont immergées en position verticale entre -10 et $-40 \mathrm{~cm}$ et le biotecton développé à la surface des lames analysé à l'issue d'une période de 6 jours, 13 jours et 26 jours. Les valeurs indiquées sur le tableau 2 sont calculées pour une profondeur moyenne d'immersion de $-25 \mathrm{~cm}$. Le C.O.T. et les pigments chlorophylliens sont calculés pour chacune des 3 séries de lames par référence à une surface totale d'échantillonnage équivalente à $75 \mathrm{~cm}^{2}$. La production primaire est estimée, en l'occurrence, par la méthode de Gaarder et Gran, par temps ensoleillé, durant le tiers médian de la journée solaire ; les lames recouvertes de biotecton sont incubées en enceintes de verre saturées en eau provenant de l'étang Petit Turlet et filtrée sur Whatman $\mathrm{GF} / \mathrm{C}$; la production brute d' $\mathrm{O}_{2}$ par photosynthèse des microphytes du biotecton est convertie en carbone assimilé en adoptant un coefficient photosynthétique de 1,2 .

Plusieurs remarques découlent de la lecture du tableau 2:

- Le taux de recouvrement des lames par le biotecton au cours des 4 semaines d'immersion traduit l'enrichissement progressif en valeur absolue (colonne 1 du tableau) et en valeur relative (colonne 3) de la charge en matières organiques du biotecton. L'accroissement du rapport C.O.T./chlorophylle traduit la diversité croissante des sources d'accumulation des matières organiques, un coefficient $\leqslant 60$, assimilable à celui calculé à l'issue de la 1 ere semaine et de la $2^{\mathbf{e}}$ semaine d'exposition des lames, étant généralement retenu pour convertir les chlorophylles mesurées en carbone organique particulaire associé à la fraction végétale vivante.

- Le rapport entre le C.O.T. et la biomasse de $N$. christinae, seule espèce de Naididae présente sur les lames, tend au bout de 26 jours vers une valeur qui est circonscrite à l'intervalle de confiance de la moyenne calculée in vitro dans l'expérience décrite en 3.2.; à l'issue d'une période d'incubation de 4 semaines.

- L'accroissement des effectifs de $N$. christinae qui représentent la biomasse principale des brouteurs du biotecton recouvrant les lames affecte en retour la biomasse et la productivité des producteurs primaires selon un processus qui s'apparente au modèle de Lamberti \& Moore 1984 (cité par Planas \& Moreau 1989) : une pression de broutage modéré accroît la production primaire par rapport à la biomasse algale au cours des 2 premières semaines ; un taux de broutage plus important entraîne, pour sa part, au cours des semaines suivantes, une réduction simultanée de la production et de la biomasse algales.

3.4.2. Un autre type d'expérience recourant à l'utilisation de substrats artificiels a été réalisé au niveau de la rivière expérimentale du Campus de l'Université Claude Bernard. Cette rivière est constituée de 2 chenaux parallèles de $40 \mathrm{~m}$ de long sur $1 \mathrm{~m}$ de large, avec une hauteur d'eau réglée en moyenne à $14 \mathrm{~cm}$, un débit de $71 . \mathrm{s}^{-1}$ et une vitesse moyenne de $6 \mathrm{~cm} \mathrm{~s}^{-1}$ (Gaudin \& Caillère 1985). Cette rivière est alimentée par pompage dans la nappe phréatique

Tableau 2. Paramètres impliqués dans le suivi du biotecton développé sur lames de verre immergées.

Etang Petit Turlet ; expérience du 10/05 au 5/06/1989.

Table 2. Parameters used in following the biotecton development on immersed glass slides. Pond Petit Turlet; experiment from May 10 to June 051989.

\begin{tabular}{|c|c|c|c|c|c|c|c|}
\hline & $\begin{array}{l}\text { C.o.T. (biatectan) } \\
\left(\mu \mathrm{cm}^{-2} \mathrm{~cm}^{-2}\right.\end{array}$ & $\begin{array}{l}\text { Chiorophylle (biot ecton) } \\
\left(\mu \mathrm{cm} . \mathrm{cm}^{2}\right)\end{array}$ & $\frac{\text { C.O.T. }}{\text { Chlorophylle }}$ & $\begin{array}{l}\text { Production primaire } \\
\text { biotecton }\left(\mu \mathrm{g} . \mathrm{C} . \mathrm{cm}^{-2} . \mathrm{h}^{-1}\right)\end{array}$ & $\frac{\text { Production primaire }}{\text { Chlorophylle }}$ & $\begin{array}{c}\text { Biomasse (Nais) } \\
\left(\mu \mathrm{g}\left(\text { poids sec). } \mathrm{cm}^{2}\right)\right.\end{array}$ & $\frac{\text { C.0.T. (biotecton) }}{\text { Biomasse (Nais) }}$ \\
\hline $\begin{array}{c}10 / 05 \rightarrow 16 / 05 \\
(6 j .)\end{array}$ & 90 & 2,86 & 31 & 7,65 & 2,67 & 1,64 & 54,8 \\
\hline $\begin{array}{c}10 / 05->23 / 05 \\
(13 \mathrm{j})\end{array}$ & 210 & 3,75 & 56 & 25,4 & 6,77 & 17,4 & 12,0 \\
\hline $\begin{array}{c}10 / 05-5 / 06 \\
(26 j)\end{array}$ & 245 & 2,52 & 97 & 3,67 & 1,45 & 35,2 & 6,96 \\
\hline
\end{tabular}


avec un débit constant de $50 \mathrm{~m}^{3} \mathrm{~h}_{-1}$. Il s'agit d'une eau bicarbonatée calcique riche en sulfates et en silice comme en témoignent les valeurs moyennes des éléments majeurs suivants (Alfaro-Tejera 1982) : $\mathrm{HCO}_{3-1}: 273 \mathrm{mgl}^{-1}, \mathrm{Ca}++: 105 \mathrm{mgl}^{-1}, \mathrm{Mg}^{++}$: $12 \mathrm{mgl}^{-1}, \mathrm{SO}_{4^{-}}: 69 \mathrm{mgl}^{-1}, \mathrm{SiO}_{2}: 12 \mathrm{mgl}^{-}, \mathrm{Cl}^{-}:$ $11 \mathrm{mgl}^{-1}$. Chacun des chenaux est relié à l'amont à un bassin permettant le brassage et l'oxygénation de l'eau et la rivière alimente en aval un étang expérimental de $800 \mathrm{~m}^{2}$.

L'expérience a consisté à déposer sur le fond de l'un des chenaux de la rivière, le 23/12/1992, 12 plaques de $15 \times 15 \mathrm{~cm}$ recouvertes de toile émeri conformes au modèle décrit en 2.2.2. Ces substrats artificiels ont été laissés en place durant 2 mois avant d'être récupérés le 20/02/1993.

Le biotecton revêtant la surface des plaques était constitué principalement, à l'issue de l'expérience, de diatomées du genre Nitzschia $(>90 \%$ de la biomasse algale estimée). Les peuplements d'invertébrés associés au biotecton étaient dominés numériquement par les Oligochètes de la famille des Naididae (228 individus en moyenne par plaque), les larves d'insectes (Chironomidae et Trichoptères, 85 individus en moyenne par plaque) et les Crustacés (Aselles, Gammares, Ostracodes, 23 individus en moyenne par plaque).

Sur le tableau 3 figurent les coefficients de corrélation entre différentes variables du compartiment producteurs primaires et du compartiment consommateurs primaires (Naididae) du biotecton, les 4 espèces de Naididae étant classées par ordre d'importance numérique : Chaetogaster diastrophus (59 \% des Naididae), N. pseudobtusa (32\%), $N$. communis $(7 \%), N$. variabilis $(2 \%)$.

Ce tableau fait état d'une corrélation significative entre le C.O.T. et la chlorophylle du biotecton ( $r$ $=0,784, p<0,01$ ), à mettre en parallèle avec la faible valeur du rapport C.O.T./chlorophylle $a \mathrm{du}$ biotecton à l'issue de la période d'immersion des substrats, soit $27,1 \pm 2,92$ valeur qui témoigne de l'activité métabolique soutenue des diatomées, composante essentielle du biotecton, le rapport moyen entre chlorophylle active et phéodérivés atteignant pour sa part la valeur relativement élevée de 5,30 $\pm 0,83$.

Tableau 3. Coefficients de corrélation entre différentes variables contrôlées à l'issue de l'expérience conduite du 23/12/1992 au 20/02/1993.

Rivière expérimentale (Campus de l'Université Claude Bernard). Les coefficients sont calculés en prenant en compte le nombre d'individus (Naididae) et le poids (en $\mathrm{mg}$ ) du biotecton en C.O.T. et en pigments chlorophylliens par plaque.

Table 3. Coefficients of correlation between variables checked at the end of the experiment from 23 December 1992 to 20 February 1993.

Experimental river (University Claude Bernard, Lyon I). The coefficients take into account the naidid densities and the T.O.C. and chlorophyll concentration (mg) per plate.

\begin{tabular}{lcc}
\hline & C.0.T. & Chlorophylle a \\
\hline $\begin{array}{l}\text { Chlorophylle a } \\
\text { Phéopigments a }\end{array}$ & $r=0,210 \mathrm{NS}$ & $r=0,344 \mathrm{NS}$ \\
$\begin{array}{l}\text { Chaetogaster diastrophus } \\
\text { Nais (toutes espèces } \\
\text { Confondues) }\end{array}$ & $r=0,224 \mathrm{NS}$ & $r=0,333 \mathrm{NS}$ \\
$\begin{array}{l}\text { Nais pseudobtusa } \\
\text { Nais communis }\end{array}$ & $r=0,590 ; p<0,05$ & $r=0,555 \mathrm{NS}$ \\
Nais variabilis. & $r=0,642 ; p<0,05$ & $r=0,607 ; p<0,05$ \\
\hline
\end{tabular}


La matrice des corrélations entre les effectifs de Naididae et les charges respectives en C.O.T. et chlorophylle ne révèle pas, en règle générale, de corrélation significative, excepté en ce qui concerne $N$. communis. C. diastrophus est classé parmi les espèces algivores sensu stricto se nourrissant préférentiellement de diatomées ; il peut paraître, en première approximation, surprenant de n'observer aucune corrélation positive significative entre les effectifs de cette population et la charge en microphytes caractérisant la structure du biotecton. Il convient toutefois de rappeler, bien que cette remarque ne préjuge pas de la nature et de l'évolution des relations entre producteurs et consommateurs primaires au cours de la période d'immersion des substrats du 23/12/1992 au 20/02/1993, que les diatomées du genre Nitzschia d'une taille moyenne de $320 \mu \mathrm{m}$ qui composaient l'essentiel de la biomasse algale du biotecton à l'issue de l'expérience ont une taille incompatible avec les capacités de broutage de C. diastrophus. D'autre part, cette espèce sélectionne les algues qui interviennent dans son alimentation, contrairement à d'autres Naididae telles que $N$. pseudobtusa, considérée comme une espèce opportuniste se nourrissant préférentiellement des algues ou autres particules comestibles les plus fréquentes à un instant donné, (Mc Elhone 1979).

\subsection{Impact des bactéries associées au périphyton sur la production des. Naididae algivores}

L'absence, en règle générale, de corrélation significative entre, d'une part, la biomasse et (ou) la production de Nais spp. et, d'autre part, la charge en microphytes des substrats nutritifs estimée par le biais des pigments chlorophylliens (cf. 3.2, 3.4.2) tend à confirmer l'éclectisme alimentaire des espèces du genre Nais, algivores sensu lato. Le rôle joué notamment par les bactéries associées aux microphytes du périphyton dans le taux de croissance de populations de Naididae a pu être démontré en comparant la production de Naididae in vitro, en présence et en l'absence d'antibiotiques.

Une expérience de ce type a été réalisée à l'emplacement de l'étang implanté en aval de la rivière expérimentale du Campus du 28/06 au 7/07/1989, en recourant à la technique des enceintes immergées in situ. Elle consistait à comparer l'évolution des populations de Nais spp. (regroupant 3 espèces, $N$. pseudobtusa, $N$. communis et $N$. christinae) mises en incubation dans 2 séries d'enceintes renfermant au départ de l'expérience 5,10 ou 15 individus et un mélange d'eau phréatique $(50 \mathrm{ml})$ et de périphyton $(20 \mathrm{mg})$ riche en diatomées en provenance de tiges submergées de Phragmites implantées dans l'étang où était réalisée l'expérience. L'une des 2 séries d'enceintes recevait au départ une injection d'antibiotiques, l'autre série servant de témoin.

Le tableau 4 rend compte de l'impact des antibiotiques sur 2 paramètres impliqués dans la dynamique des populations asexuées de Nais spp. : le taux d'accroissement instantané $r=1 / \Delta t . \log _{e} N_{t} / N_{0}$ (où $\mathrm{N}_{0}$ est le nombre de zoïdes parentaux introduits dans chaque enceinte au temps $t_{0}$ et $N_{t}$ le nombre de zoïdes parentaux à l'issue de l'expérience de 9 jours), et d'autre part, le taux moyen de stolonisation (S), correspondant à la valeur moyenne du rapport entre le nombre de stolons et le nombre de zoïdes parentaux isolés ou en chaîne.

La figure 2 offre une illustration de l'influence des divers types de conditionnement (présence ou absence d'antibiotiques, nombre de spécimens de Nais mis en incubation) sur le taux d'accroissement instantané des populations. L'expérience montre que r est réduit de $19,0 \%, 21,3 \%$ et $20,7 \%$, avec respectivement 5,10 et 15 zoïdes parentaux au départ de l'expérience, dans les enceintes soumises à l'injection d'antibiotiques, par rapport aux enceintes témoins.

Une réduction significative des taux d'accroissement, dans les proportions de $19,0 \%$ et $31,4 \%$ (enceintes témoins) et de $21,3 \%$ et $32,9 \%$ (enceintes soumises aux antibiotiques) est également observée pour des effectifs de départ, respectivement de 10 et 15 zoïdes parentaux, par référence aux populations dérivant d'un stock initial de 5 zoïdes. Cette réduction qui affecte des populations pour lesquelles le rapport C.O.T. (substrat)/biomasse (Nais) oscille, en fin d'expérience, entre les valeurs de 4,6 et 2,1 témoigne d'un effet de densité-dépendance (cf. 3.3).

La réduction observée au niveau de la productivité des populations de Naididae se reproduisant par paratomie, perçue comme la conséquence de la stérilisation du substrat nutritif, n'est pas comparable à l'impact d'antibiotiques sur des communautés de protozoaires inféodés au biofilm de filtres à écoulement au compte-goutte de stations de traitement d'eaux polluées (Stanislawska 1979). Selon cet auteur, une injection de streptomycine de concentration 
Tableau 4. Paramètres impliqués dans l'étude d'impact d'injection d'antibiotiques. Etang expérimental (Campus de l'Université Claude Bernard) ; expérience du 28/06 au $7 / 07 / 1989$.

Les deux dernières colonnes du tableau concernent la charge en chlorophylle $a$ et en phéodérivés dans le périphyton de chaque enceinte à l'issue de l'expérience. $\mathrm{t}=$ enceintes témoins abritant un stock initial de 5,10 ou 15 zoïdes parentaux. $\mathrm{a}=$ enceintes soumises à l'injection d'antibiotiques et abritant un stock initial de 5, 10 ou 15 zoïdes parentaux. r : taux d'accroissement instantané (Nais spp.). $\mathrm{s}=$ taux moyen de stolonisation (Nais spp.).

Table 4. Parameters analyzed with regard to the impact of antibiotics. Experimental pond (University Claude Bernard, Lyon I).

The two last columns of the table refer to the chlorophyll and pheopigments a (in $\mathrm{mg} \mathrm{g}^{-1}$ periphyton) in each enclosure at the end of the experiment.

$t=$ control enclosures with 5,10 or 15 parental zooïds at the start ot the experiment $-\mathrm{a}=$ enclosures sterilized by injection of antibiotics with 5, 10 or 15 parental zooids at the start of the experiment. $\mathrm{r}=$ daily growth rate (Nais spp.). $\mathrm{s}=$ mean stolonization rate (Nais spp.).

\begin{tabular}{|c|c|c|c|c|}
\hline conditionnements & $r$ & 5 & $\begin{array}{c}\text { Chlorophylle a } \\
+ \text { phéodérivés } \\
(\mu \mathrm{g} \cdot \mathrm{g}-1)\end{array}$ & $\frac{\text { Phéopigments a }}{\text { Chlorophylle a }}$ \\
\hline$t_{5}$ & 0,289 & 1,06 & 1422 & 0,91 \\
\hline tro & 0,234 & 0,82 & 1682 & 0,90 \\
\hline$t_{15}$ & 0,198 & 0,67 & 1324 & 1,27 \\
\hline as & 0,234 & 0,82 & 1847 & 1,61 \\
\hline$a_{10}$ & 0,184 & 0,59 & 1962 & 1,25 \\
\hline$a_{15}$ & 0,157 & 0,57 & 2078 & 1,27 \\
\hline
\end{tabular}

proche de celle utilisée dans cette expérience (15 $\left.\mathrm{mgl}^{-1}\right)$ entraîne une disparition complète des protozoaires consommateurs de bactéries saprophytes avant la régénération complète des communautés 30 jours après l'apport d'antibiotiques.

Par ailleurs, la température moyenne, relevée à l'emplacement des enceintes durant l'expérience, soit $18,7^{\circ} \mathrm{C}$, est proche de la température de $20^{\circ} \mathrm{C}$ à laquelle un effet significatif sur la croissance des Naididae en milieux de culture enrichis en bactéries était observé en conditions expérimentales (Lochhead \& Learner 1983).

En accord avec Berland et Maestrini (1969), on constate que de faibles adjonctions d'antibiotiques tendent à favoriser la croissance des diatomées, comme le montrent les concentrations en chlorophylle $a$ et en phéodérivés du périphyton à l'issue de l'expérience dans les enceintes ayant reçu une injection d'antibiotiques. Cette surcharge en microphytes ne s'accompagne pas d'un effet secondaire favorable sur la production de Naididae en milieu carencé en bactéries, ce qui tend à confirmer le rôle important des bactéries associées aux microphytes du périphyton dans l'alimentation des organismes brouteurs du périphyton. Le parallélisme (cf. fig. 2) entre les 2 séries de mesure des taux d'accroissement (respectivement dans les enceintes avec antibiotiques et les enceintes témoins), que confirme un coefficient de corrélation significatif $(\mathrm{r}=0,998$, $\mathrm{p}<0,05$ ) entre les valeurs de chaque couple de données en fonction des 3 types de conditionnement biologique, apporte également la confirmation indirecte de l'influence déterminante d'une carence du milieu nutritif en bactéries sur la croissance des populations de Naididae.

\section{Conclusions}

Cette étude consacrée au biotecton et conçue en terme de bilan trophique témoigne de l'intérêt respectif du carbone organique et des Naididae pour appréhender les relations de nature trophique entre 


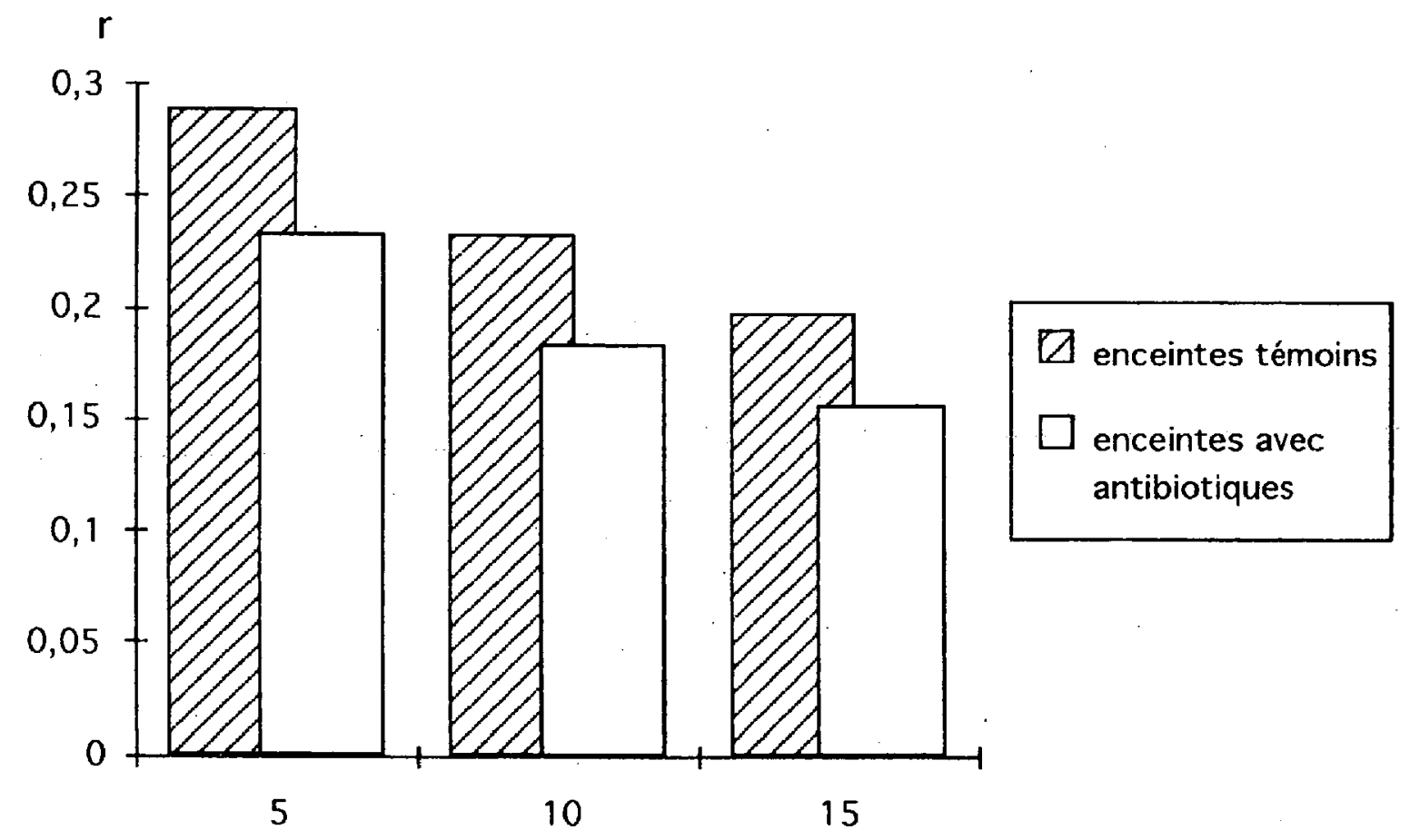

Fig. 2. Taux d'accroissement instantanés (r) de populations de Nais spp., correspondant à un stock initial de 5 , 10 et 15 zoïdes parentaux, incubés en enceintes soumises ou non à l'action d'antibiotiques.

Fig. 2. Daily growth rates (r) applicable to populations of Nais spp. as a function of the biological conditioning (cultivation of 5,10 or 15 parental zooids in experimental enclosures, with and without antibiotics).

producteurs et consommateurs de matières organiques. En qualité de cause et conséquence de l'activité biologique, l'élément carbone peut servir de traceur chimique aussi bien pour évaluer les potentialités énergétiques d'un substrat nutritif et calculer les flux de matière et d'énergie au sein d'un réseau trophique que pour déceler d'éventuels dysfonctionnements consécutifs, par exemple, au surpeuplement. Le C.O.T. est un outil conventionnel de mesure de la charge globale en matières organiques d'un substrat nutritif tel que le biotecton. Mais il peut également, à ce titre, servir d'élément de référence pour évaluer l'importance relative et l'activité biologique des microphytes considérés en tant que source potentielle de nourriture pour les Naididae. Le C.O.T. peut également servir d'élément de contrôle pour tester la présence ou l'absence et évaluer l'importance de relations de densité-dépendance au sein du biotecton. Le caractère opportuniste du régime alimentaire et des stratégies de reproduction prédispose, pour sa part, les Naididae et notamment le genre Nais, à servir de matériel biologique, pour étudier, en conditions expérimentales, le fonctionnement du biotecton. Le recours aux divers protocoles expérimentaux pratiqués à l'occasion de cette étude tend à confirmer le régime alimentaire mixte des Naididae dont la productivité est favorisée à la fois par l'accroissement de la charge globale en matières organiques et l'accroissement de la diversité en ressources trophiques de leur environnement. L'expérimentation décrite en 3.5 confirme, à cet égard, le rôle déterminant des bactéries dans l'alimentation de ces organismes qui ne sont algivores qu'en apparence, conformément à l'opinion de nombreux auteurs (Learner \& al. 1978, Harper et al. 1981a, b, Lochhead \& Learner 1983, Mc Cullough \& Jackson 1985). A titre d'indicateurs biologiques du niveau trophique et de la qualité des eaux et des sédiments (Lafont 1989), des représentants de la famille des Naididae, conjointement à ceux d'autres familles d'Oligochètes aquatiques, peuvent également servir à la mise au point de tests expérimentaux 
de toxicité (Chapman \& Mitchell 1981, Smith et al. 1991). La complémentarité des protocoles expérimentaux, tels que l'expérimentation in vitro et in situ pratiqués à l'occasion de ce type d'études, devrait permettre, à cet égard, de minimiser les risques consécutifs à l'extrapolation des résultats expérimentaux aux conditions naturelles.

Compte tenu des résultats obtenus, à l'occasion de ce bilan, certaines voies de recherches mériteraient d'être plus particulièrement explorées. L'étude, applicable aux hydrosystèmes fluviaux, de l'influence de la variabilité de l'habitat à différentes échelles d'espace et de temps sur la dynamique de trois composantes du biotecton; les microphytes, les bactéries et les Naididae, constitue un type d'approche susceptible de confrontation avec des modèles théoriques tels que le « Patch Dynamics Concept 》 (Townsend, 1989) et le "River Habitat Templet Concept " (Resh et al., 1994). Perçue comme la résultante de trois types d'activités en étroite connexion (production primaire des microphytes, production bactérienne hétérotrophe, production secondaire des consommateurs algivores et bactériophages), la biologie du biotecton peut être aussi appréhendée en termes de rendement de production et de rendement écologique. Dans le cas particulier des biofilms développés à l'interface eau-sédiment, la mesure du rapport entre, d'une part, la production des Naididae et, d'autre part, la production des microphytes et des bactéries représente un objectif potentiel de recherche qui s'apparente aux recherches conduites récemment dans une annexe fluviale du Rhône (Martinet et al. 1993) en vue de comparer la production nette des Tubificidae, Oligochètes endobenthiques, consommateurs de sédiments, et le taux d'accumulation (ou production nette) des sédiments.

\section{Travaux cités}

Abrahamsen G. 1973. - Studies on body-volume, body-surface area, density and live weight of Enchytraeidae (Oligochaeta). Pedobiologia, 13 : 6-15.

Alfaro-Tejera L. 1982. - Dynamique de population de l'espèce Armiger crista (L.), Mollusque Planorbidae. Thèse $3^{\mathrm{C}}$ cycle, Université Claude Bernard (Lyon I), $106 \mathrm{p}$.

Amino A. 1983. - Dosage de la chlorophylle et des phéopigments par spectrophotométrie. In CNEXO ed. Manuel des analyses chimiques en milieu marin, pp 177-192.

Berland B. \& Maestrini S. 1969. - Action de quelques antibiotiques sur le développement de 5 diatomées en culture. $J$. Expl. Mar. Biol. Ecol., 3 : 62-75.
Chapman P.M. \& Mitchell D.G. 1986. - Acute tolerance tests with the oligochaetes Nais communis (Naididae) and Ilyodrilus frantzi (Tubificidae). Hydrobiologia 137 : 61-64.

Gaudin P. \& Caillère L. 1985. - Relations chabots-truites : résultats obtenus en rivière expérimentale. Verh. Internat. Verein. Limnol., 22 : 2581-2586.

Giani N. 1984. - Contribution à l'étude de la faune d'eau douce et plus particulièrement des oligochètes. II. Les oligochètes aquatiques : taxinomie, répartition et écologie. Thèse, Université Paul Sabatier, Toulouse, 196 p.

Harper R.M., Fry J.C. \& Learner M.A. 1981a. - A bacteriological investigation to elucidate the feeding biology of Nais variabilis (Oligochaeta Naididae). Freshwat. Biol., 11 : 227-236.

Harper R.M., Fry J.C. \& Learner M.A. 1981b. - Digestion of bacteria by Nais variabilis (Oligochacta) as established by autoradiography. Oikos $36: 211-218$.

Juget J. 1979. - La texture granulométrique des sédiments et le régime alimentaire des Oligochètes limnicoles. Hydrobiologia 65 : 144-154.

Juget J. 1984. - Oligochaeta of the epigean and underground fauna of the alluvial plain of the French upper Rhône (biotypological trial). Hydrobiologia 115 : 175-182.

Juget J. \& Rostan J.C. 1973. - Influence des herbiers à Trapa natans sur la dynamique d'un étang en période estivale. Annls Limnol., 9 : 11-23.

Juget J., Goubier V. \& Barthelemy D. 1989. - Intrinsic and extrinsic variables controlling the productivity of asexual populations of Nais spp Naididae, Oligochaeta). Hydrobiologia, $180: 177-184$.

Juget J. \& Lafont M. 1994 _ - Theoretical habitat templets, species traits, and species richness : aquatic Oligochaetes in the Upper Rhône River and its floodplain. In B. Statzner, V.H. Resh \& S. Doledec, eds. Ecology of the Upper Rhône River : a test of habitat templet theories. Freshwat. Biol. 31 : 327-340.

Lafont M. 1989. - Contribution à la gestion des eaux continentales : utilisation des Oligochètes comme descripteurs de l'état biologique et du degré de pollution des eaux et des sédiments. Thèse de Doctorat d'Etat ès Sciences. UCBL Lyon I : $311 \mathrm{p}$ + annexes 92 p.

Lamberti G.A. \& Moore J.W. 1984. - Aquatic insects as primary consumers. In : (V.H. Resh \& D.M. Rosenberg (eds). The Ecology of Aquatic Insects. Praeger Special StudiesPraeger Scientific. New York, pp 164-195.

Learner M.A., Lochhead G. \& Hughes B.D. 1978. - A review of the biology of British Naididae (Oligochaeta) with emphasis on the lotic environment. Freshwat. Biol., $8: 357-375$.

Lellouch J. \& Lazar P. 1974. - Méthodes statistiques en expérimentation biologique. Flammarion, Paris, 283 p.

Lochhead G. \& Learner M.A. 1983. - The effect of temperature on asexual population growth of three species of Naididae (Oligochaeta). Hydrobiologia, 98 : 107-112.

Loden M.S. 1981. - Reproductive ecology of Naididae (Oligochaeta). Hydrobiologia, 83 : 115-123.

Lorenzen C.J. 1967. - Determination of chlorophyll and phaeopigments : spectrophotometric equations. Limnol. Oceanogr., $12: 343-346$.

Martinet F., Juget J. \& Riera P. 1993. - Carbon fluxes across water, sediment and benthos along a gradient of disturbance intensity : adaptive responses of the sediment feeders. Arch. Hydrobiol., 127 : 39-56. 
Mc Cullough J.D. \& Jackson D.W. 1985. - Composition and productivity of the benthic macroinvertebrate community of a subtropical reservoir. Int. Rev. ges. Hydrobiol., 70 : 221-235.

Mc Elhone M.J. 1979. - A comparison of the gut contents of two co-existing lake-dwelling Naididae (Oligochaeta), Nais pseudobtusa and Chaetogaster diastrophus. Freshwat. Biol., $9: 199-204$

Mc Elhone M.J. 1980. - Some factors influencing the diet of coexisting benthic algal grazing Naididae (Oligochaeta). Can. J. Zool., $58: 481-487$.

Planas D. \& Moreau G. 1989. - Interaction périphyton-benthos en milieu acidifié. Revue des sciences de l'Eau, 2 : 607-619.

Provot M. 1982. - Aspects écophysiologiques des relations entre le phytobenthos (Diatomées) et les Naididae (Oligochètes). Rapport DEA, Université Claude Bernard (Lyon I), 46 p. Resh V.H., Hildrew A.G., Statzner B. \& Townsend C.R. 1994. - Theoretical habitat templets, species traits, and species richness : a synthesis of long-term ecological research on the Upper Rhône River in the context of concurrently developed ecological theory. In B. Statzner, V.H. Resh \& S. Doledec, eds. Ecology of the Upper Rhône River : a test of habitat templet theories. Freshwat. Biol., 31 : 539-554.

Riera P., Juget J. \& Martinet F. 1991 . - Predator-prey interactions : effects of carp predation on tubificid dynamics and carp production in experimental fishpond. Hydrobiologia, $226: 129-136$.

Smith D.P., Kennedy J.H. \& Dickson K.L. 1991. - An evaluation of a Naidid Oligochaete as a toxicity test organism. Environ. Toxicol. Chem., $10: 1459-1465$.

Stanislawska J. 1979. - Communities of organisms during treatment of sewage containing antibiotics. Pol. Arch. Hydrobial., $26: 221-229$.

Townsend C.R. 1989. - The patch dynamics concept of stream community ecology. J.N. Am. benthol. Soc., $8: 36-50$. 